\title{
Producción científica en enfermería transcultural en el periodo 2007-2014
}

Scientific production in Transcultural Nursing during the period 2007-2014

Produção científica de enfermagem transcultural no período 2007-2014

Fátima Berchid-Martínez¹, Raquel Herrero-Hahn², César Hueso-Montoro ${ }^{3}$

${ }^{1}$ Graduada en enfermería.

${ }^{2}$ Departamento de Enfermería, Facultad de Ciencias de la Salud, Universidad de Granada.

${ }^{3}$ PhD. Departamento de Enfermería, Facultad de Ciencias de la Salud. Universidad de Jaén.

Cómo citar este artículo en edición digital: Berchid-Martínez, F., Herrero-Hahn, R., \& Hueso-Montoro, C. (2017).

Producción científica en enfermería transcultural en el periodo 2007 - 2014. Cultura de los Cuidados (Edición digital), 21(49).

Recuperado de http://dx.doi.org/10.14198/cuid.2017.49.13

Correspondencia: remitirse al correo electrónico.

Correo electrónico: fatima.berchid@gmail.com

Recibido: 08/03/2017; Aceptado: 04/06/2017

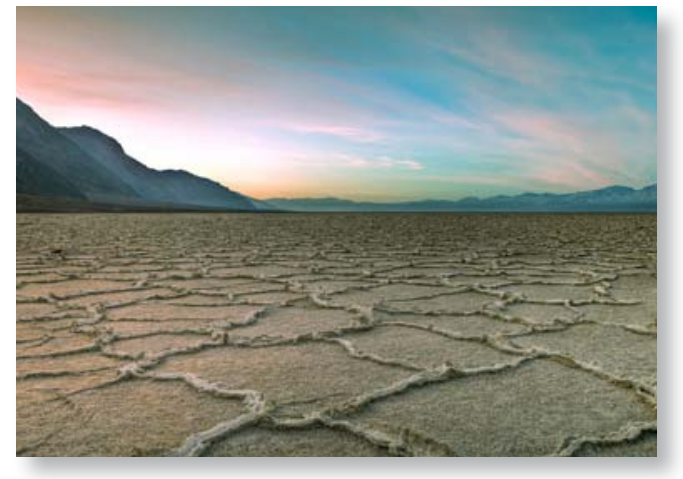

ABSTRACT

The objective was to identify and analyze the scientific production on Transcultural Nursing in the international context in the period between 2007 and 2014.

A literature review on the CINAHL and CUIDEN database was performed using the descriptor "Transcultural Nursing" and including only original or research articles published between 2007 and 2014. A documentary descriptive study of the selected literature was carried out using bibliometric indicators.

A total of 155 articles were obtained. The profile of the publications is the one of works signed by 2 or three women authors who mainly belong to the nursing discipline and whose work reference institution is the university. Most of these studies were conducted in the United States, Brazil and Spain. They are fundamentally qualitative design studies that address as major thematic the experiences and beliefs regarding health or disease, the training of students and professionals, and their proceeding in the field of cultural diversity.

Key words: Transcultural nursing, culture, review literature as topic, bibliometrics, review.

\section{RESUMEN}

El objetivo fue identificar y analizar la producción científica sobre Enfermería Transcultural en el contexto internacional en el periodo de tiempo comprendido entre los años 2007 y 2014.

Se realizó una revisión bibliográfica en las bases de datos CINAHL y CUIDEN empleando el descriptor "Enfermería Transcultural" e incluyendo exclusivamente artículos origina- 
les o de investigación publicados entre 2007 y 2014. Se llevó a cabo un estudio documental descriptivo sobre la bibliografía seleccionada utilizando indicadores bibliométricos.

Se obtuvieron un total de 155 artículos. El perfil de las publicaciones es el de trabajos firmados por entre 2 y 3 autores, mujeres, pertenecientes principalmente a la disciplina enfermera y cuya institución de referencia es la universidad. La mayoría de estos trabajos se realizaron en Estados Unidos, Brasil y España. Los hallazgos señalan que los artículos encontrados se derivaron principalmente de estudios cualitativos que abordaban temas como las experiencias y creencias ante la salud o la enfermedad, la formación de estudiantes y profesionales, y el abordaje de los mismos en el ámbito de la diversidad cultural.

Palabras clave: Enfermería transcultural, cultura, literatura de revisión como asunto, bibliometría, revisión.

\section{RESUMO}

O objetivo foi identificar e analisar a produção científica sobre Enfermagem Transcultural no contexto internacional no período entre 2007 e 2014.

Foi realizada uma revisão da literatura na base de dados CINAHL e CUIDEN utilizando o descritor "Enfermagem Transcultural" e incluindo únicamente pesquisas originais ou de investigação publicados entre 2007 e 2014 . A metodología foi estruturada como um estudio descritivo documental da literatura selecionada por meio de indicadores bibliomètricos.

Foi obtido um total de 155 artigos. Os resultados mostram que as publicações foram feitas por entre 2 e três mulheres autores que pertencem principalmente à disciplina de enfermagem e cuja instituição de referência de trabalho é a universidade. A maioria destes estudos foram conduzidos nos Estados Unidos, Brasil e Espanha. Os artigos encontrados foram derivados de estudos qualitativos sobre questões como experiências e crenças sobre saúde ou doença, a formação de estudantes e profissionais e seu processo no campo da diversidade cultural.

Palavras-chave: Transcultural enfermagem, cultura, literatura de revisão como assunto, bibliometria, revisão.

\section{INTRODUCCIÓN}

El concepto de cultura surge en Alemania a fínales del siglo XVIII (Hidalgo, 2005). A partir de ese momento son muchos los autores que proponen una definición para este constructo. Sin embargo, en el ámbito de la enfermería, el planteado por Leininger (1991) parece ser uno de los más aceptados y empleados, quién la define como el conjunto de valores, creencias, normas y modos de vivir compartidos, aprendidos y transmitidos que guían los pensamientos, las decisiones y las acciones de un grupo de manera específica.

En general, debido a la mundialización y al aumento de las migraciones y los intercambios internacionales, la diversidad cultural es un hecho, conduciendo a la integración de diversos intercambios multiculturales en casi todos los contextos nacionales, lo cual corre a la par con la tendencia hacia filiaciones culturales múltiples y una complejidad creciente de las identidades culturales (UNESCO, 2010).

En el ámbito de la salud, esta realidad multicultural conlleva la necesidad de conocer las condiciones de las personas contextualizándolas en su propia cultura, buscando así entender su perspectiva y forma de interpretar la salud $y$ la enfermedad para brindar un cuidado de enfermería eficaz, satisfactorio y coherente con su cultura. 
El campo de la Enfermería Transcultural surgió como respuesta a la necesidad de implementar modelos que adoptasen enfoques antropológicos y considerasen el factor cultural e ideológico como objeto de estudio (Siles et al. 2001).

\section{Teorías de Enfermería Transcultural}

La Dra. Madeleine Leininger fundó durante los años cincuenta el campo de la Enfermería Transcultural, que definió como el área formal de estudio y trabajo centrado en el cuidado basado en la cultura, creencias de salud o enfermedad, valores y prácticas de las personas, para ayudarlas a mantener o recuperar su salud o bienestar, afrontar su enfermedad, su discapacidad o hacer frente a su muerte (Leininger, 1991, 1994).

Cada individuo o grupo interpreta desde su propia perspectiva cultural (construida por experiencias, creencias, valores, mitos, etc.) las situaciones de salud, enfermedad, dolor y muerte (Ginzburg, citado por Siles, 2008). En este sentido, el cuidado enfermero, desde la Enfermería Transcultural, consiste en entender la diversidad de significados e implicaciones que tiene el cuidado de la salud para las diferentes culturas, y dentro de éstas, para cada individuo; y de esta forma, entender a la persona como un ser único para poder brindarle un cuidado individualizado y adaptado culturalmente.

Partiendo de lo anterior, se plantea realizar una revisión bibliográfica con el objetivo de identificar y analizar la producción científica generada en el campo de la Enfermería Transcultural en el periodo 2007 - 2014. Se pretende mostrar las tendencias en investigación durante estos años, así como analizar el comportamiento bibliométrico de la misma.

\section{METODOLOGÍA}

Se realizó un estudio documental descriptivo a partir de una revisión bibliográfica.

\section{Material de estudio}

Se seleccionaron artículos originales o de investigación centrados en el campo de la Enfermería Transcultural y publicados entre 2007 y 2014. La búsqueda se realizó en el periodo comprendido entre enero y abril de 2015 con objeto de recopilar todos los artículos publicados en el año 2014. Se excluyeron aquellas publicaciones originales del tipo "creación/validación de cuestionarios" cuyo objetivo era la validación transcultural de instrumentos clínicos, ya que se alejaban de la temática central del estudio.

\section{Recogida de datos}

Se seleccionaron las bases de datos CINAHL y CUIDEN debido a su especialización en el ámbito de la enfermería y a que, entre ambas, se cubre un área geográfica importante en cuanto a producción científica internacional se refiere. Cabe suponer que las revistas indexadas en estas bases de datos dan cuenta de la práctica totalidad de la producción científica en enfermería. Estudios previos avalan esta decisión; tal es el caso del estudio de Murphy (2006), cuyo objetivo fue analizar las bases de datos con el acceso más completo a la literatura en Enfermería Transcultural, mostrando a CINAHL como una de las bases de datos con más amplia cobertura.

Se utilizó el descriptor MeSH “Transcultural Nursing" para la búsqueda en CINHAL y "Enfermería Transcultural" en CUIDEN. Se utilizó este único descriptor por la amplitud y a la vez concreción del mismo en relación al objetivo del estudio. En ambas bases de datos se utilizaron los filtros disponibles para delimitar la búsqueda a artículos originales o de investigación y limitados al periodo de tiempo determinado previamente, con objeto de aplicar los criterios de inclusión antes descritos. 
Para la recogida de datos se realizó una primera búsqueda en ambas bases de datos en enero de 2015 y una segunda búsqueda en abril de 2015. Se revisaron los títulos y resúmenes de los registros proporcionados, se excluyeron los estudios que no cumplían los criterios de inclusión y se eliminaron los registros duplicados encontrados en ambas bases de datos. Posteriormente se llevó a cabo la lectura de los artículos, extrayendo la información necesaria para dar respuesta al objetivo del estudio.

\section{Indicadores}

Los datos que se recogieron fueron: número de autores por artículo, sexo del autor principal, institución de trabajo del autor principal, área de conocimiento del autor principal, colaboración interdisciplinar, país de procedencia do para las variables cualitativas tablas de frecuencia y porcentajes, y para las variables cuantitativas medidas de tendencia central y de dispersión. Posteriormente se hizo un análisis del contenido de las publicaciones seleccionadas, siendo agrupadas en diferentes categorías temáticas.

\section{RESULTADOS Y DISCUSIÓN}

Se obtuvieron un total de 271 resultados distribuidos en 94 (34,7\%) en CUIDEN y 177 $(65,3 \%)$ en CINAHL. Tras aplicar los criterios de inclusión y exclusión, se obtuvieron 155 documentos, lo que supone un 57,6\% de la muestra inicial. Éstos se distribuyeron en 128 $(82,1 \%)$ en CINAHL y $27(21,1 \%)$ en CUIDEN. En la figura 1 se representa el diagrama de búsqueda y selección de documentos. del autor principal, colaboración internacional, número de revistas, número de publicaciones por año, población de estudio, diseño del estudio, contenido del estudio.

\section{Análisis de datos}

Se asignó un número de identificación a cada registro ingresado en la base de datos creada para la recogida de la información.

Para el análisis estadístico se empleó el programa IBM SPSS 22.0 para Windows. Para el estudio bibliométrico se utilizó la estadística descriptiva, emplean-
Figura 1. Diagrama de flujo

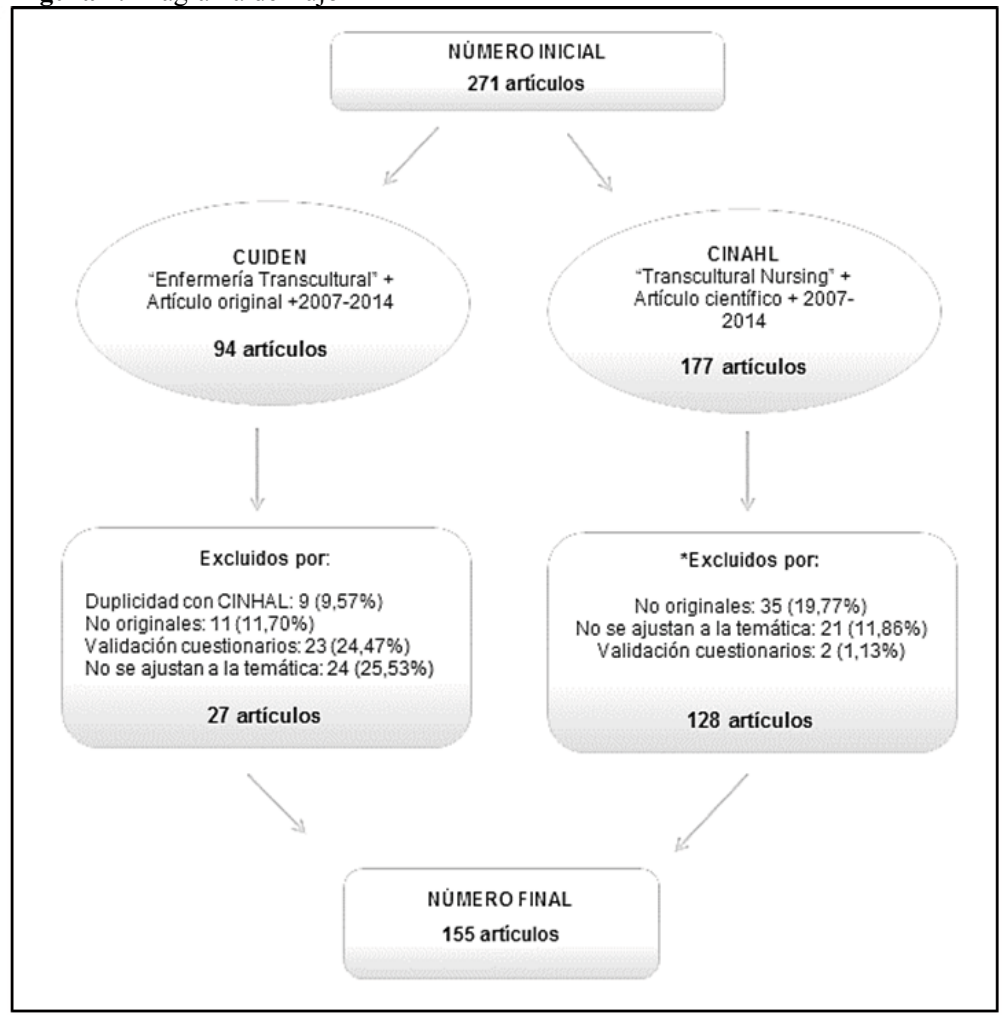

*El $n \square$ mero de art culos finalmente obtenidos de CINAHL presentan un error de registro entre lo inicialmente encontrados y los finalmente seleccionados del 5,08\%. 
El número medio de autores por artículo fue de 2,9 ( $\mathrm{DE} \pm 1,79$ ), con un rango de 1-10, siendo más frecuentes publicaciones realizadas entre 2 y 3 autores, número que queda lejos de cifras que marcan cierta consolidación del trabajo en equipo, teniendo en cuenta que los grupos de investigación competitivos suelen constituirse por un mínimo de 6 investigadores.

Se han observado diferencias importantes en cuanto al sexo, encontrando que la mayoría de trabajos son liderados por mujeres (83,9\%). Si a ello se le une que la mayor representación en cuanto a ámbito de conocimiento lo tiene enfermería, se puede concluir que el perfil mostrado en las publicaciones en este campo coincide con el perfil profesional de la disciplina enfermera, ligada históricamente a las mujeres (García, Sainz \& Botella, 2004).

Respecto a la institución de trabajo del autor principal, $118(76,1 \%)$ trabajaba en la universidad, 19 (12,3\%) en una institución de salud, 10 $(6,5 \%)$ en centros de investigación y $8(5,1 \%)$ en otro tipo de instituciones. El hecho de que la universidad destaque como la institución con mayor producción científica es coherente y reconoce el papel clave de la institución académica no sólo como transmisora de conocimiento sino también como generadora del mismo.

En lo que se refiere al área de conocimiento del autor principal, se hallaron autores pertenecientes a 8 disciplinas diferentes (Tabla 1). Además de enfermería, que es la más representativa (58,7\%), aparecen otros ámbitos de conocimiento, entre los que cabe destacar la antropología. También es importante el número de publicaciones de autores con ambas titulaciones, lo cual se puede deber al ámbito de estudio de esta revisión. La simbiosis entre estas dos disciplinas permite vertebrar la teoría y la praxis de los cuidados culturales. A este respecto, Tarrés (2001) reivindica la importancia de unir la práctica enfermera con el conocimiento antropológico con el objeto de ofrecer un mejor cuidado a una población cada vez más diversa culturalmente.

Tabla 1. rea de conocimiento del primer autor

\begin{tabular}{lc}
\hline \multicolumn{1}{c}{ mbito de conocimiento } & n (\%) \\
\hline Enfermer a & $91(58,7)$ \\
\hline Educaci $\curvearrowleft \mathrm{n}$ & $2(1,3)$ \\
\hline Enfermer a y antropolog a & $2(1,3)$ \\
\hline Epidemiolog a & $2(1,3)$ \\
\hline Medicina & $1(, 6)$ \\
\hline Nutrici $\curvearrowleft$ y diet tica & $1(, 6)$ \\
\hline Servicios humanos y trabajo social & $1(, 6)$ \\
\hline Estudiantes & $1(, 6)$ \\
\hline No identificado & $54(34,8)$ \\
\hline
\end{tabular}

Fuente. Datos obtenidos a partir de la realizaci $\square$ n de este estudio

Con respecto a la colaboración interdisciplinar de los autores se observó presencia de la misma en 34 (21,9\%) casos. En 32 (20,6\%) casos no hubo colaboración entre profesionales de diferentes ámbitos de conocimiento, y en 89 $(57,4 \%)$ no fue posible averiguar si ésta existió o no. En relación a la colaboración internacional entre autores, en $66(42,6 \%)$ publicaciones no se encontró evidencia de la misma. En 15 $(9,7 \%)$ publicaciones se observó que existía esta colaboración, y en 74 (47,7\%) casos no fue posible determinar si ésta existía o no.

Los autores principales procedían de un total de 30 países diferentes, encontrando 15 $(9,7 \%)$ casos en los que no fue posible determinar el país de origen. En mayor medida, los autores principales procedían de Estados Unidos (21,9\%), seguido de Brasil (10,3\%) y España (9,7\%), encontrando que los países anglosajones suman el 38\% de la producción en Enfermería Transcultural. Estos resultados concuerdan con los obtenidos por Juvé et al. (2011) quienes encontraron que los países anglosajones son los principales productores de conocimiento científico en enfermería. 
Las publicaciones se distribuyeron en un total de 81 revistas, siendo la que presentaba un mayor número de las mismas la Journal of Transcultural Nursing (18,7\%), revista especializada en la publicación de resultados de investigaciones que tienen implicaciones directas para la prestación de una asistencia sanitaria culturalmente competente.

Con respecto al número de publicaciones por año, éstas variaron entre 30 publicaciones en el 2007 y 11 en el año 2013. En el gráfico 1 se encuentra el porcentaje de publicaciones por año.

Gr fico 1. Distribuci $\llbracket$ n de publicaciones por a o

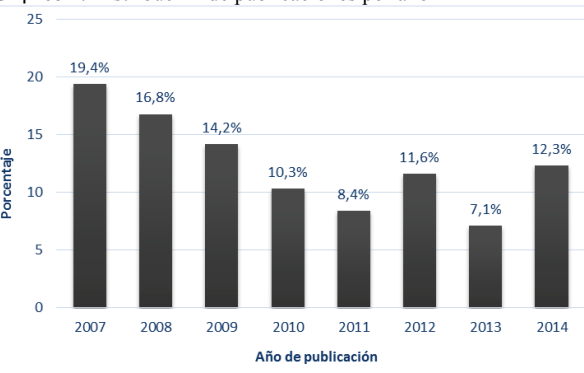

Fuente. Datos obtenidos a partir de la realizaci $\square$ de este estudio

En relación a la población objeto de estudio, es importante destacar que en la mayoría de las publicaciones ésta estuvo constituida por profesionales que, en general, son un grupo de población de interés para la investigación enfermera (Juvé et al., 2011). La tabla 2 muestra la distribución de la población de estudio de los trabaios seleccionados.

Tabla 2. Distribuci $\square$ de publicaciones por poblaci $\square \mathrm{n}$ de estudio

\begin{tabular}{|c|c|}
\hline Poblaci $n$ de estudio & n (\%) \\
\hline Profesionales & $50(32,3)$ \\
\hline Poblaci $\curvearrowleft \mathrm{n}$ general & $32(20,6)$ \\
\hline Estudiantes & $23(14,8)$ \\
\hline Pacientes & $19(12,3)$ \\
\hline Profesionales, poblaci $\mathrm{n}$ general & $11(7,1)$ \\
\hline Profesionales, estudiantes & $7(4,5)$ \\
\hline Profesionales, pacientes & $7(4,5)$ \\
\hline Otros & $3(1,9)$ \\
\hline Pacientes, poblaci $\_$general & $2(1,3)$ \\
\hline Pacientes, estudiantes & $1(, 6)$ \\
\hline
\end{tabular}

Fuente. Datos obtenidos a partir de la realizaci $\square$ de este estudio
En cuanto a la distribución de las publicaciones por diseño de estudio, 108 (69,7\%) correspondieron a diseños cualitativos, 43 $(17,7 \%)$ fueron estudios cuantitativos y 4 $(2,6 \%)$ eran a estudios de creación y validación de cuestionarios sobre constructos relacionados con la Enfermería Transcultural. El predominio de estudios de diseño cualitativo observado puede estar relacionado con el aumento en la producción de este tipo de estudios que se está produciendo en los últimos años. En este sentido, se constata lo que Amezcua y Carricondo (2000) vaticinaban cuando afirmaban que existe una relación directa entre las estrategias de humanización de la salud y la adopción de metodologías cualitativas, por lo que cabe esperar que éstas se generalicen en la medida en que el cuidado del paciente se aborde desde una perspectiva más holística. Por tanto, si consideramos la cultura como un elemento clave a tener en cuenta en el cuidado integral del paciente, se asume que la metodología cualitativa es la que mejor responde a preguntas de investigación que surjan desde esta perspectiva sociocultural.

Con respecto al análisis de contenido realizado, los estudios se pueden clasificar en tres categorías temáticas: experiencias y creencias ante la salud o la enfermedad desde una perspectiva transcultural, formación de los profesionales y estudiantes en enfermería transcultural y abordaje del cuidado transcultural por parte de los profesionales.

\section{Experiencias y creencias ante la salud o la enfermedad desde una perspectiva transcul- tural}

Diversos estudios muestran el papel de la enfermería transcultural en el cuidado de la salud y la enfermedad. En esta línea se sitúa el estudio de Díaz et al. (2011), quienes afirman 
que el reconocimiento, fortalecimiento y recuperación de las prácticas de cuidado propias de cada grupo cultural mejora la calidad del cuidado de enfermería.

El estudio de creencias en salud y enfermedad desde una perspectiva cultural se evidencia en trabajos que tratan de recuperar estos elementos en grupos delimitados culturalmente (Wehbe-Alamah, 2011; Gentil, 2012; Penaranda \& Romero, 2010); estudios en situaciones de cuidado específicas o ante enfermedades concretas (Bernardes, Rangel \& Meneses, 2009; Castellanos \& Lucero, 2012; Pennafort, Queioz \& Joge, 2012) y trabajos interesados en la singularidad de determinados subgrupos (García-Navarro \& Gualda, 2014).

\section{Formación de los profesionales y estudiantes de ciencias de la salud}

La formación de los profesionales y estudiantes de ciencias de la salud y su adquisición de competencia cultural es una preocupación presente en el campo de la Enfermería Transcultural, encontrando que los mismos profesionales manifiestan las preocupaciones y necesidades de formación que tienen para poder proporcionar un cuidado cultualmente congruente con la cultura de las personas (Tuohy, McCarthy, Cassidy \& Graham, 2008).

En esta línea, Pértega, López, Hospital y Baonza (2010) sugieren que acciones de entrenamiento en Enfermería Transcultural pueden ayudar a contrarrestar las actitudes etnocéntricas que se pueden presentar en los profesionales de la salud y que afectan a la calidad del cuidado que se proporciona. En este sentido, varios estudios sostienen que es el profesorado universitario el que debe mostrar al estudiante la necesidad de proporcionar un cuidado cultualmente competente y ofrecerle las herramientas necesarias para ello (Allen,
Brown, Duff, Nesbitt \& Hepner, 2013; Gebru \& William, 2010; Merrell, Olumine \& Khanom, 2014).

Por otro lado, se hallan estudios que sostienen que el mejor modo de que una persona pueda adquirir competencia cultura es mediante la exposición cultural (Bohman \& Borglin, 2014; Jirwe, Gerrish \& Emami, 2010).

\section{Abordaje del cuidado transcultural por par- te de los profesionales}

Los profesionales encuentran barreras de diversa índole a la hora de proporcionar cuidados a personas diversas culturalmente que en muchos casos desencadenan situaciones de estrés, preocupación y miedo a la hora de afrontar la práctica profesional (Chang, Yang \& Kuo, 2013; Debesay, Harslof, Rechel \& Vike, 2014). Estas situaciones estresantes vividas por los profesionales les llevan a cuestionarse la eficacia y la calidad de los cuidados, lo cual en muchos casos se traduce en insatisfacción profesional (Allensworth-Davies et al., 2007).

A pesar de que hay estudios que revelan actitudes etnocéntricas por parte del personal sanitario, otros muestran que hay profesionales que adoptan una actitud positiva ante la diversidad cultural, tratando de adaptar el cuidado a la cultura de las personas (Allan, 2010), y manifestando la necesidad de elaborar protocolos sanitarios que consideren los aspectos culturales (Álvarez, 2014).

Entre las limitaciones de la revisión, conviene señalar que, a pesar de haber empleado un descriptor amplio, es probable que muchos estudios sobre el fenómeno de la cultura y el cuidado no se hayan localizado con la ecuación de búsqueda empleada. Quizás se deberían haber empleado otros descriptores como competencia cultural o diversidad cultural, que en bases de datos específicas como las emplea- 
das podrían haber arrojado más resultados de interés en torno el objeto de la revisión. Habría que comprobar si aumenta considerablemente el resultado, de ser así se podría pensar en un posible sesgo de indexación, ya que el descriptor empleado debiera estar presente en cualquier estudio relacionado con la cultura y los cuidados. En cualquier caso, los resultados de este estudio, sin ser concluyentes, sí muestran tendencias en relación a la producción científica en Enfermería Transcultural.

\section{CONCLUSIONES}

La producción científica en Enfermería Transcultural, en lo que respecta a producción de conocimiento primario, se caracteriza por trabajos elaborados entre 2 y 3 autores, mujeres, pertenecientes principalmente a la disciplina enfermera y cuya institución de referencia es la Universidad. La mayoría de estos trabajos se realizan en Estados Unidos, destacando también Brasil y España entre los principales países productores. Son estudios con diseño cualitativo principalmente y que abordan como temáticas principales las experiencias y creencias ante la salud o la enfermedad desde una perspectiva transcultural, la formación de los profesionales y estudiantes en enfermería transcultural y el abordaje del cuidado transcultural por parte de los profesionales.

\section{BIBLIOGRAFÍA:}

- Allan, H. (2010). Mentoring overseas nurses: Barriers to effective and non-discriminatory mentoring practices. Nursing Ethics, 17 (5), 603-13. doi: 10.1177/0969733010368747

- Allen, J., Brown, L., Duff, C., Nesbitt, P., Hepner, A. (2013). Development and evaluation of a teaching and learning approach in cross-cultural care and antidiscrimination in university nursing students. Nurse Education Today, 33 (12), 1592-1598. doi:10.1016/j. nedt.2012.12.006

- Allensworth-Davies, D., Leigh, J., Pukstas, K., Geron, S.M., Hardt, E., Brandeis, G... Parker, V.A. (2007). Country of origin and racio-ethnicity: are there differen- ces in perceived organizational cultural competency and job satisfaction among nursing assistants in long-term care? Health Care Manage, 32 (4), 321-9. Recuperado de http://www.ncbi.nlm.nih.gov/pubmed/18075441

- Álvarez, M.C. (2014). La diversidad cultural. Una realidad en las unidades de alto riesgo. Hygia de Enfermería, 85, 27-36. Recuperado de http://www.colegioenfermeriasevilla.es/wp-content/uploads/Hygia85.pdf

- Amezcua, M., Carricondo, A. (2000). Investigación Cualitativa en España. Análisis de la producción bibliográfica en salud. Index de Enfermería, 10 (28-29), 26-34. Recuperado de http://www.index-f.com/indexenfermeria/28-29revista/28-29_articulo_26-34.php

- Bernardes, M.D., Rangel, L., Meneses, I.M. (2009). Maternal care in the management of child asthma: the contributions of transcultural nursing. Escola Anna Nery, 13 (4), 772-779. Recuperado de http://dx.doi.org/10.1590/ S1414-81452009000400012

- Bohman, D.M., Borglin, G. (2014). Student exchange for nursing students: Does it raise cultural awarness'? A descriptive, qualitative study. Nurse Education In Practice, 14, 259-264. doi: 10.1016/j.nepr.2013.11.006

- Castellanos, F., Lucero, A. (2012). Prácticas religiosas en un grupo de personas mayores en situación de discapacidad y pobreza. Revista Investigación en Enfermería: Imagen y Desarrollo, 14 (2), 51-61. Recuperado de http:// www.redalyc.org/articulo.oa?id=145226758004

- Chang, H.Y., Yang, Y.M., Kuo, Y.L. (2013). Cultural sensitivity and related factors among community health nurses. Journal of Nursing Research, 21 (1), 67-73. doi: 10.1097/jnr.0b013e3182829cb9

- Debesay, J., Harslof, I., Rechel, B., Vike, H. (2014). Facing diversity under institutional constraints: challenging situations for comunity nurses when providing care to ethnic minority patients. Journal of Advanced Nursing, 70 (9), 2107-2016. doi: 10.1111/jan.12369

- Díaz, O.S., Callejas, M., Camargo, P.J., Correal, I.J., Cruz, F.T., Hernández, A.C... Pacheco, A.J. (2011). Cosmovisión ancestral y comprensión de los conceptos salud, cuidado y enfermería en habitantes del resguardo muisca de Chía, 2010. Revista Colombiana de Enfermería, 6 (6), 35-46. Recuperado de http://www.uelbosque.edu. $\mathrm{co} /$ sites/default/files/publicaciones/revistas/revista_colombiana_enfermeria/volumen6/cosmovision_ancestral.pdf

- García, A. M., Sainz, A., Botella, M. (2004). La enfermería vista desde el género. Index de Enfermería, 13 (46), 45-48. Recuperado de http:// scielo.isciii.es/scielo.php?script=sci_arttext\&pid $=$ S1132-12962004000200009

- García-Navarro, B., Gualda, E. (2014). Cuidado transcultural y estrategias familiares ante la dependencia: el fenómeno de los cuidadores extranjeros. Aquichan, 14 (4), 509- 522. doi: 10.5294/aqui.2014.14.4.6

- Gebru, K., William, A. (2010). Education to promote culturally competent nursing care-a content anaysis of students responses. Nurse Education Today, 30 (1), 54- 
60. doi: 10.1016/j.nedt.2009.06.005

- Gentil, I. (2012). Worldview of Sub-Saharan emigrants and nursing care. Index de Enfermería, 21 (3), 121125. Recuperado de http://dx.doi.org/10.4321/S113212962012000200003

- Hidalgo, V. (2005). Cultura, Multiculturadidad, Interculturalidad: Evolución del término. Ciències de l'Educació, 20, 73-84. Recuperado de http://www.fec-chiapas.com. $\mathrm{mx} /$ sistema/biblioteca_digital/cultura-multiculturalidad-interculturalidad-y-transculturalidad.pdf

- Jirwe, M., Gerrish, K., Emami, A. (2010). Student nurses' experiences of communication in cross-cultural care encounters. Scandinavian Journal of Caring Sciences, 24 (3), 436-44. doi: 10.1111/j.1471-6712.2009.00733.x

- Juvé, M.E., Pastor, L., Estrem, M.M., Blanco, C., Verge, J.M., Coiduras, A...Lizarralde, J.P. (2011). ¿De qué se ocupan las enfermeras? Estudio transversal de la producción científica reciente. Nursing, 29 (10), 5659. Recuperado de http://diposit.ub.edu/dspace/bitstream/2445/34080/1/615390.pdf

- Leininger, M. (1991). Culture Care Diversity \& Universality: A Theory of Nursing. New York: National League for Nursing Press.

- Leiniger, M. (1994). Transcultural Nursing: Concepts, theories and practices. Ohio: Greyden Press.

- Merrell, J., Olumine, G., Khanom, A. (2014). Working in progress': nurse educators' views on preparing preregistration nursing students en Wales for practice in multiethnic enviroments. Journal of Research in Nursing, 19 (6), 490-501. DOI: 10.1177/1744987114546723

- Murphy, S.C. (2006). Mapping the literature of transcultural nursing. Medical Library Association, 94 (supl. 2), 143-151. Recuperado de http://www.ncbi.nlm.nih. gov/pmc/articles/PMC1463039/pdf/i1536-5050-09402S-0143.pdf

- Penaranda, J.A., Romero, A. (2010). Conservación y adaptación de los cuidados culturales en salud de los Wiwa, en Colombia. Memorias, 8 (14), 9-23. Recuperado de http://revistas.ucc.edu.co/index.php/me/article/ view/162/163

- Pennafort, V.P., Queiroz, M.V., Jorge, M.S. (2012). Children and adolescents with chronic kidney disease in an educational-therapeutic enviroment: support for cultural nursing. Revista da Escola de Enfermagem, 46 (5), 1057-1065. Recuperado de http://dx.doi.org/10.1590/ S0080-62342012000500004

- Pértega, E.M., López, M., Hospital, A., Baonza, S. (2010). Effectiveness of a formative program about transcultural nursing on aspects of the mental health on inmigrants children between 12 and 17 years old diagnosed of stress for movement sindrome. Nure Investigación, 7 (44). Recuperado de https://doaj.org/article/26289eae9a8a4c208 5131320c99ea667

- Siles, J., Cibanal, L., Vizcaya, F., Gabaldón, E., Domínguez, J.M., Solano, C. \& García, E. (2001, 2º semestre). Una mirada a la situación científica de dos especialidades esenciales de la enfermería contemporánea: la An- tropología de los Cuidados y la Enfermería Transcultural. Cultura de los Cuidados, 5 (10), 72 - 87. Recuperado de http://rua.ua.es/dspace/handle/10045/4938

- Siles, J. (2008, 2º semestre). Historia de la enfermería: una aportación epistemológica desde la perspectiva cultural de los cuidados. Cultura de los Cuidados, 12 (24): 5 - 6. Recuperado de http://rua.ua.es/dspace/hand$\underline{\text { le/10045/9862 }}$

- Tarrés, S. (2001). El cuidado del 'otro'. Diversidad cultural y enfermería transcultural. Gazeta de Antropología, 17. Recuperado de http://www.gazeta-antropologia. $\mathrm{es} / \mathrm{p}=3267$

- Tuohy, D., McCarthy, J., Cassidy, I., Graham, M.M. (2008). Educational needs of nurses when nursing people of a different culture in Ireland. International Nursing Review, 55 (2), 164-170. doi: 10.1111/j.14667657.2007.00600.x

- UNESCO (Organización de las Naciones Unidas para la Educación, la Ciencia y la Cultura). (2010). Informe Mundial de la UNESCO. Invertir en la diversidad cultural y el diálogo intercultural. París: Organización de las Naciones Unidas para la Educación, la Ciencia y la Cultura.

- Wehbe-Alamah, H. (2011). The use of culture care theory with Syrian muslims in the mid-western United States. Online Journal of Cultural Competence in Nursing and Healthcare, 1 (3), 1-12. Recuperado de http://ojccnh.org/pdf/v1n3al.pdf 